\title{
The Progression of Heart Rate Variability Parameters Throughout Cardiac Rehabilitation
}

\author{
Hélène De Cannière ${ }^{1,2}$, John Morales ${ }^{4}$, Carolina Varon ${ }^{4}$, Christophe JP Smeets ${ }^{1,2,3}$, Melanie Schoutteten ${ }^{1,2}$, \\ Chris Van Hoof ${ }^{4,6}$, Willemijn Groenendaal ${ }^{3}$, Sabine Van Huffel ${ }^{4}$, Pieter Vandervoort ${ }^{1,2,5}$ \\ ${ }^{1}$ Mobile Health Unit, Faculty of Life Sciences, Hasselt University, Belgium \\ ${ }^{2}$ Future Health Department, Ziekenhuis Oost-Limburg, Belgium \\ ${ }^{3}$ Holst Centre/imec Netherlands, The Netherlands \\ ${ }^{4}$ KU Leuven, Department of Electrical Engineering (ESAT), STADIUS Center for Dynamical \\ Systems, Signal Processing and Data Analytics, Leuven, Belgium \\ ${ }^{5}$ Department of Cardiology, Ziekenhuis Oost-Limburg, Belgium \\ ${ }^{6}$ imec vwz Belgium, Belgium
}

\begin{abstract}
To optimize exercise management in cardiac patients following a cardiac rehabilitation $(C R)$ program, it is important to study short-term changes of parameters, representative for exercise capacity. We hypothesize that a detailed study of these parameters could provide more insight into the actual improvement of the patients. Therefore, the progression of heart rate variability (HRV) parameters during short-term intervals throughout $C R$ was investigated in this study.

Electrocardiographic (ECG) signals, recorded with a wearable device in 129 patients following a CR program, were analyzed. Patients participated in a follow up protocol. Analyses on the normalized power in the low frequency band $(L F n)$ and the root mean square of successive differences (RMSSD) were performed for patients on and off drug therapy.

Although no significant differences were found, treating CR patients with ACE-inhibition or beta blockers (BB) tended to have an influence on the HRV parameters. The progression of the HRV parameters throughout CR was mostly characterized by a non-monotonic trend. These insights elucidate the changes occurring in the regulatory mechanisms. Moreover, findings of this work give new valuable insights for the close monitoring of disease progression during $C R$ in future applications.
\end{abstract}

\section{Introduction}

Increasing evidence suggests an imbalance of the autonomic nervous system as a biomarker for a broad spectrum of cardiac diseases. Studying these alterations in patients with cardiovascular problems might provide more insight into the actual disease progression [1-3]. A noninvasive tool to quantify autonomic activity and its alterations is the analysis of heart rate variability (HRV). The HRV represents the variation in the time intervals between consecutive heartbeats. The HRV can be characterized using different measures in the time and frequency domain. Vagal activity is often quantified by the root mean square of the successive differences (RMSSD) in the time domain, while both low frequency (LF) and high frequency (HF) have been used in the frequency domain to assess the different branches of the autonomic nervous system. The HF power is often associated with vagal activity, while LF has been shown to reflect both vagal and sympathetic activity. It is generally accepted that a decline in the HRV parameters that quantify vagal activity are associated with a worse outcome [1-4]. However, when assessing the HRV, a number of confounding factors should be considered. In a patient population, not only disease type and severity, but also intervention, i.e. drug therapy or rehabilitation can have confounding effects.

Previous studies showed that clinical interventions within a cardiac rehabilitation (CR) population can induce an increase in parasympathetic activity and a decrease in sympathetic activity [5-9]. This is reflected both in the time and in the frequency domain parameters derived from the HRV. Both ACE-inhibition and beta-blocker (BB) treatment strategies have been suggested to improve the regulatory mechanism of HRV in populations with cardiac problems. Similarly, the combination of medication with exercise interventions showed a decrease in sympathetic activity and an increase in parasympathetic activity [1012]. These confounding factors are characteristic for a $C R$ patient population and need to be considered when interpreting the progression of HRV parameters 
throughout the CR program.

In this study, we investigated the change of HRV parameters in cardiac patients with reduced ejection fraction (EF), during their rehabilitation program. To study the short-term changes, measurements were performed every three weeks during a three-month rehabilitation program. More specifically, the effect of drug therapy on the response of HRV to rehabilitation was studied.

\section{Material and methods}

\subsection{Patients}

One hundred nineteen cardiovascular patients, participating in a structured multidisciplinary CR program in a single tertiary care centre (Ziekenhuis Oost-Limburg, Genk, Belgium), were included. Mean age was $63 \pm 1$ years and $71 \%$ of patients were male. Baseline mean EF was 47 $(35-55) \%$, and $55 \%$ of the patients had ischemic cardiomyopathy. $76 \%$ of subjects were on angiotensinconverting-enzyme inhibitor (ACE) therapy; $75 \%$ were on beta blocker (BB) therapy, and $61 \%$ of these patients were taking both. To study the effects of ACE-therapy, the complete patient population was divided into two groups, taking ACE inhibitors and not taking ACE inhibitors. Similarly, to investigate BB treatment, all patients were divided into the $\mathrm{BB}$-group and the non-BB group. It is not excluded that patients on ACE-inhibition therapy are also treated with $\mathrm{BB}$. On the other hand, patients not treated with BB can also be on ACE-inhibition treatment. The study complied with the Declaration of Helsinki and the local institutional committee approved the study protocol. Written informed consent was obtained from all subjects.

\subsection{Cardiac Rehabilitation}

After a cardiovascular-related hospitalization or consultation, patients were referred to the multidisciplinary CR program. Enrolled patients followed 45 sessions of supervised ambulatory rehabilitation at a frequency of three one-hour-sessions a week. The training protocol consisted of both aerobic and resistive exercises. The supervising physiotherapist increased the training intensity based on individual performance every two weeks.

\subsection{Experiments}

A six-minute walking test (6MWT) was performed at baseline (i.e. start of the rehabilitation program) and was repeated four times, every three weeks, resulting in a total of five measurements. The 6MWT was performed according to a standardized protocol [13]. Preceding the 6MWT, patients were at rest for five minutes to obtain their resting heart rate. Afterwards, a 5-minute recuperation phase followed the 6MWT. During the complete protocol (five minutes of rest, 6MWT and five minutes of recuperation) patients were equipped with a wearable device (imec, Eindhoven), which was used to record ECG signals at a sampling frequency of $512 \mathrm{~Hz}$.

\subsection{Parameter extraction}

The signals were divided into the segments belonging to each of the phases described in section 2.3. At the transition from resting to walking and from walking to recuperation, 10 seconds of ECG signal were removed, so that transient effects on the HRV were excluded. An initial detection of R-peaks was done automatically with the algorithm and tool described in [14] and [15], next wrong detections were visually corrected. The Integral Pulse Frequency Modulation (IPFM) model was used to build an HRV representation and to mitigate the effect of ectopic beats on the HRV parameters [16]. HF power (HF: 0.15$.040 \mathrm{~Hz}$ ), LF power (LF: 0.04-0.15 Hz) and RMSSD values were calculated for each phase, resulting in three HRV values per patient per session. HF and LF powers were normalized (HFn and LFn respectively) and thereby represented the relative value of each power component in proportion to the total power in the band between 0.03 and $0.4 \mathrm{~Hz}$ [2]. Moreover, the difference in HRV between the different phases of activity and inactivity could contain additional information on progression throughout the CR program. Therefore, to study these changes, the differences in HRV $, \Delta_{\text {rest-walk }}, \Delta_{\text {walk-recup }}, \Delta_{\text {rest-recup }}$ were respectively calculated. Next, the effect of drug therapy on the change of these HRV parameters and their delta differences throughout the CR program were studied.

\subsection{Statistics}

Normality was tested using the Shapiro-Wilk statistics. A two-way mixed ANOVA investigated the effect of ACEinhibition and $\mathrm{BB}$ treatment on the progression of HRV parameters throughout a $\mathrm{CR}$ program. When significant, a univariate procedure was performed to analyse the simple main effect for medication intake. Next, a repeated measures ANOVA was performed to analyse the simple main effect over time (across five measurement sessions). The same tests were performed on the delta differences between the different phases to study the effect of medication intake on the progression throughout CR. The statistical significance was set at a 2-tailed probability level of $<0.05$. Statistics were performed using SPSS version 24 (IBM, Chicago, Illinois).

\section{Results and discussion}

The effect of ACE-inhibition or BB treatment on the progression of HRV parameters throughout CR was not statistically significant for any phase. This was tested when 

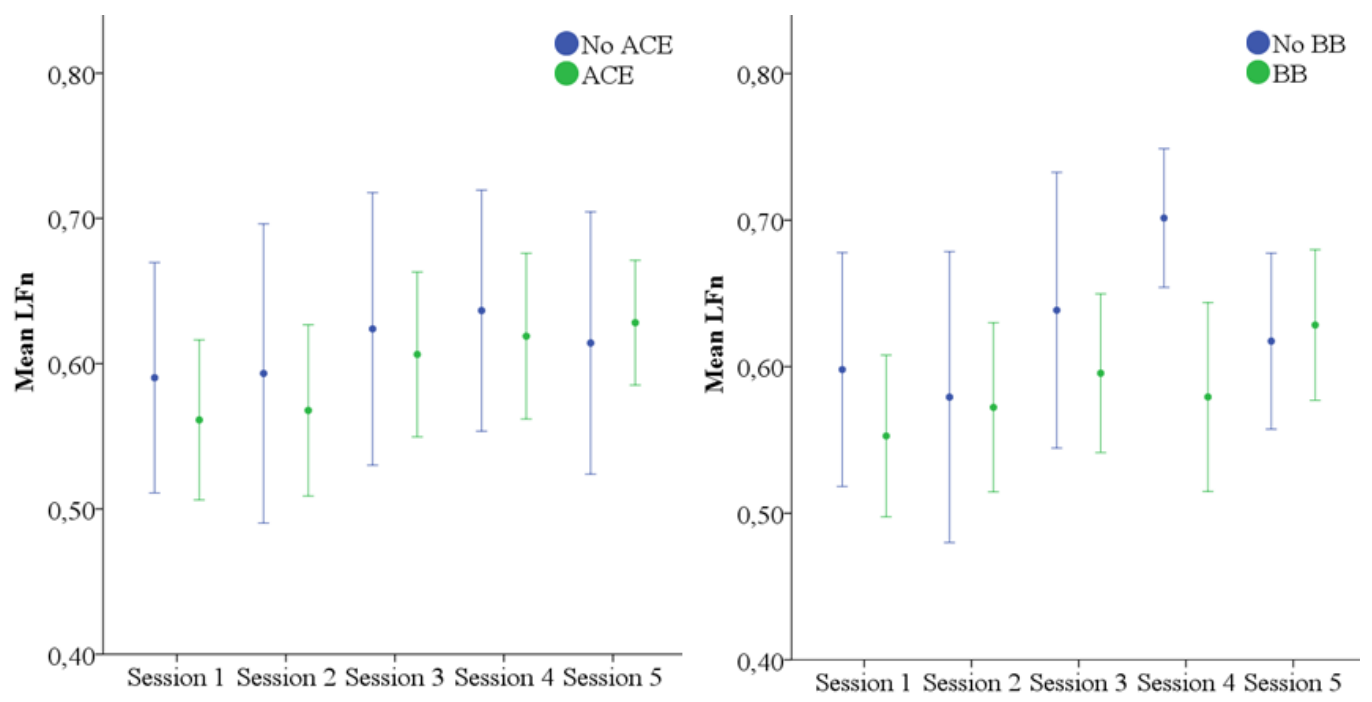

Figure 1 Clustered error bar of LFn for every session throughout CR for ACE inhibition (a) and BB treatment (b) in recuperation phase.

comparing the intake of the drugs over time for the LFn, HFn, LF, HF and RMSSD. Nevertheless, some trends were observed in the data. Firstly, the effect of ACE-inhibition or $\mathrm{BB}$ treatment throughout $\mathrm{CR}$ on the progression of LFn during the recuperation phase was investigated (Figure 1). An increasing trend in LFn was seen for all groups. Although small and not significant, the increase tended to be higher in patients on ACE-inhibition compared to patients not taking ACE-inhibition (ACE: difference between mean LFn session 5 and session 1=0.07; NoACE: difference between mean LFn session 5 and session $1=0.02$ ). Patients not taking BB showed an increase up until the fourth session followed by a drop in LFn power during the final session. Overall, when only comparing start and end of CR, LFn showed a very small increase for the non-BB group compared to the BB-group (BB: difference between mean LFn session 5 and $1=0.02$; NoBB: difference between mean LFn session 5 and $1=0.08$ ).

Malfatto et al. showed a decrease in LFn power for patients following CR. This effect was amplified in patients taking BB [17]. These results are opposite to our results in which both the non-BB and BB-group showed an increase in LFn. A possible explanation could be the treatment strategy of cardiac patients. Most patients (61\%) were on a combination of BB and ACE-inhibition therapy. For analysis purpose, a distinction was only made between patients taking or not taking BB and patients taking or not taking ACE-inhibitors. Therefore, patients on a combination therapy were included in both the BB and ACE-inhibition analysis and thus it was not excluded that patients on ACE-inhibition therapy were also treated with BB. On the other hand, patients not treated with BB could also be on ACE-inhibition treatment, which could explain the similar increase in LFn for the non-BB group as for the ACE group. These confounding factors were a limitation to our study results. Moreover, a CR population consists of cardiac patients with different pathologies, i.e. heart failure and acute myocardial infarction patients, which could also have a potential influence on the results. Additionally, the ECG signals in this dataset contain many ectopic beats, affecting the computation of HRV parameters. This effect was minimized by using the IPFM model, but it is not discarded that the ectopic beats have an important confounding effect on the calculations.

Next, the effect of BB-therapy and exercise training on the progression during the resting phase was investigated (Figure 2). An increasing trend in RMSSD was seen up until the fourth session for patients on BB therapy, followed by a drop during the final session. The patients not treated with $\mathrm{BB}$, showed a non-monotonic trend in

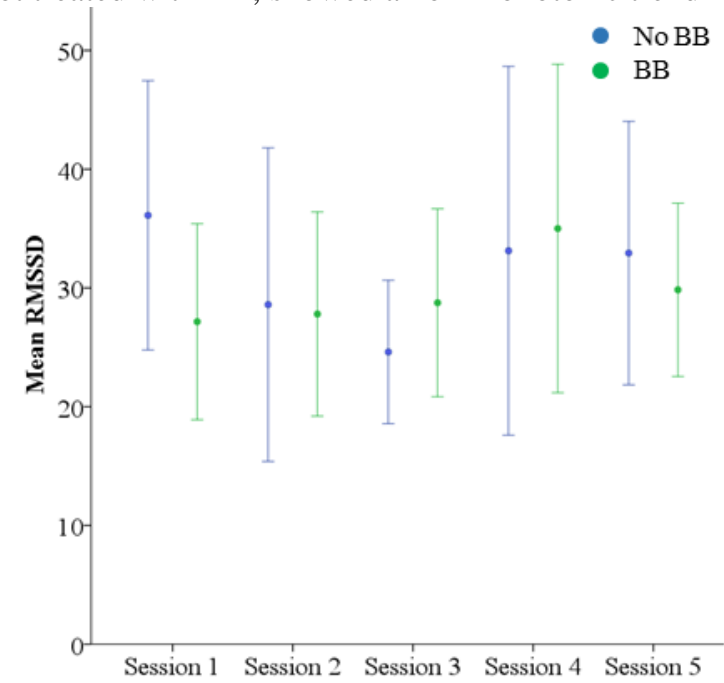

Figure 2. Clustered error bar of RMSSD for every session throughout cardiac rehabilitation for $\mathrm{BB}$ in resting phase. 
RMSSD across sessions. This result suggested that treatment with $\mathrm{BB}$, could stabilize the progression of RMSSD.

In a final step, the changes that occurred in RMSSD when shifting from the resting phase to the walking phase were examined (Figure 3). A similar trend was seen for both the $\mathrm{BB}$ and non-BB group.

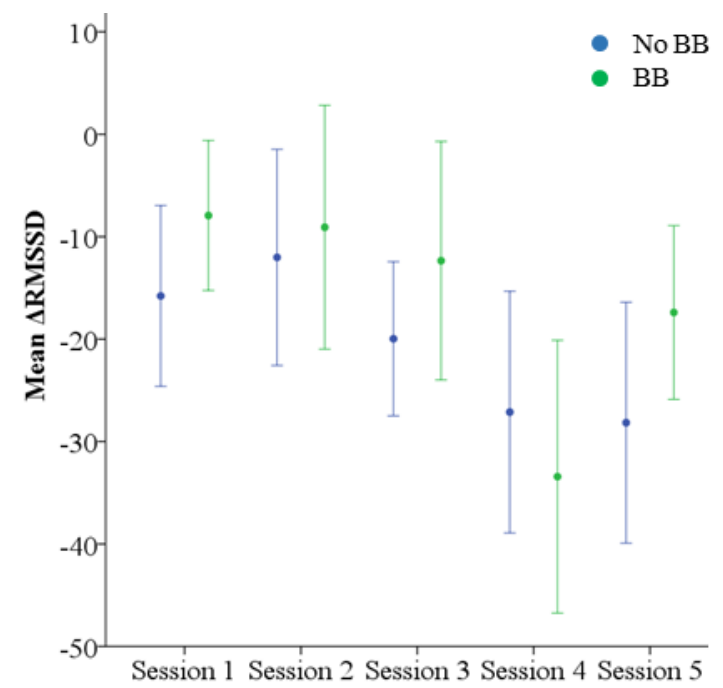

Figure 3. Clustered error bar of $\triangle \mathrm{RMSSD}$ for every session throughout cardiac rehabilitation for $\Delta_{\text {rest-walk }}$ with BB treatment.

The difference in RMSSD tended to be larger in patients not treated with BB for every session throughout CR, except for the fourth session, although not significant. This suggests a more stable vagal modulation when changing activities in the treated group. However, the disease severity in patients who were treated with BB was more pronounced, which was reflected in the ability of the autonomic nervous system to adapt to changes.

\section{Conclusion}

Treating CR patients with ACE-inhibition or BB tended to have an influence on the HRV parameters. The progression of the HRV parameters throughout CR was mostly characterized by a non-monotonic trend. These insights clarify the changes occurring in the regulatory mechanisms. Moreover, findings of this work give new insights valuable for closely monitoring of disease progression during CR in future applications. However, the complex treatment strategy and presence of ectopic beats, both confounding factors, should be taken into account when interpreting the results of the study.

\section{Acknowledgments}

This research is part of the Limburg Clinical Research Center (LCRC) UHasselt-ZOL-Jessa, supported by the foundation Limburg Sterk Merk, province of Limburg, Flemish government, Hasselt University, Jessa Hospital and Ziekenhuis Oost-Limburg. Drs. Hélène De Cannière is supported by a doctoral fellowship by the Research Foundation, Flanders, Belgium (FWO, grant number: 1S53616N). Carolina Varon is a postdoctoral fellow of the Research Foundation-Flanders (FWO).

\section{References}

[1] Thayer, JF., et al. "The relationship of autonomic imbalance, heart rate variability and cardiovascular disease risk factors." Int $\mathrm{J}$ Cardiol. 141.2 (2010): 122-31.

[2] Task Force. "Standards of measurement, physiological interpretation, and clinical use. Task Force of the European Society of Cardiology and the North American Society of Pacing and Electrophysiology." Circulation 93.5 (1996): 1043-1065

[3] Van Bilsen, M., et al. "The autonomic nervous system as a therapeutic target in heart failure: a scientific position statement from the Translational Research Committee of the Heart Failure Association of the European Society of Cardiology.” Eur. J. Heart Fail 19.11 (2017): 1361-1378

[4] Michael, S., et al. "Cardiac autonomic responses during exercise and post exercise recovery using heart rate variability and systolic time intervals- A Review.” Front. Physiol. 8 (2017): 301.

[5] Santos-Hiss, MD., et al. "Effects of progressive exercise during phase 1 cardiac rehabilitation on the heart rate variability of patients with acute myocardial infarction.” Disabil. Rehabil. 33.10(2011): 83542.

[6] Tygesen, H. et al. "Intensive home-based exercise training in cardiac rehabilitation increases exercise capacity and heart rate variability." Int. J. Cardiol. 79.2-3 (2001): 175-82.

[7] Bryniarski, L., et al. "Effect of exercise rehabilitation on heart rate variability in hypertensives after myocardial infarction." J. Hypertens. 15.12 (1997): 1739-43.

[8] Jancik, J., et al. "Heart rate variability in patients with heart failure: effect of exercise training." Scripta Medica. 77.5-6 (2004): 283-290.

[9] Murad, K., et al. "Exercise training improves heart rate variability in older patients with heart failure: a randomized controlled, Single blinded trial." Congest. Heart Fail. 18.4 (2012): 192-197.

[10] Kontopoulos, AG., et al. "Effect of angiotensin-converting enzyme inhibitors on the power spectrum of heart rate variability in postmyocardial infarction patients." Coron. Artery Dis. 8.8-9 (1997): $517-24$

[11] Badilini, F., et al. "Frequency-domain heart rate variability in 24hour holter recordings: role of spectral method to assess circadian patterns and pharmacological autonomic modulation." J. Electrocardiol. 33.2 (2000): 147-157.

[12] Jansson, K., et al. "The effects of metropolol and captopril on heart rate variability in patients with idiopathic dilated cardiomyopathy." Clin. Cardiol. 22.6 (1999): 397-402.

[13] ATS statement: guidelines for the six-minute walk test. American journal of respiratory and critical care medicine.2002;166(1):111-7.

[14] Varon, C., et al. "A novel algorithm for the automatic detection of sleep apnea from single-lead ECG.” IEEE Trans. on Biom. Eng. 62.9 (2015): 2269-2278.

[15] Moeyersons, J., et al. "R-DECO: An open-source Matlab based graphical user interface for the detection and correction of R-peaks." Biorxiv.

[16] Mateo, J. and Laguna, P. "Analysis of Heart Rate Variability in the Presence of Ectopic Beats Using the Heart Timing Signal." IEEE Trans. on Biom. Eng? 50.3 (2003): 334-43.

[17] Malfattp, G., et al. "Effects of cardiac rehabilitation and beta-blocker therapy on heart rate variability after first acute myocardial infarction.” Am. J. Cardiol. 81.7 (1198): 834-40.

Address for correspondence:

Hélène De Cannière.

Schiepse Bos 6, 3600 Genk, Belgium.

helene.decanniere@uhasselt.be 